\title{
Editorial
}

\section{Absolute, attributable, and relative risk in the management of coronary heart disease}

\begin{abstract}
Absolute risk
Treatment decisions in the prevention and management of coronary heart disease (CHD) often require knowledge about the level of risk. Risk is defined as the probability of encountering a particular event. Information about risk is obtained from cohort studies or other longitudinal studies such as randomised controlled trials. In these studies, risk is simply the incidence of the event in a particular group. For example, the risk (or probability) of death associated with coronary angiography is $0.1 \%$ (or 1 in 1000). ${ }^{1}$ This is termed the absolute risk.

In order to inform clinical decisions, it is often useful to compare the absolute risk in two or more groups having different exposures or different treatments (including treatment versus no treatment). The two main measures we use when comparing risk are attributable risk (risk difference) and relative risk (risk ratio).
\end{abstract}

\section{Attributable risk}

Attributable risk measures the excess risk accounted for by exposure to a particular factor. ${ }^{2}$ This is simply the difference between the absolute risks in the two groups. The term attributable risk is most commonly used in epidemiological studies. In the clinical environment, when comparing a particular treatment with placebo for example, the excess risk associated with treatment (attributable risk) may well be negative, if the treatment is beneficial. This is therefore termed an absolute risk reduction. Absolute risk reduction is increasingly used when reporting clinical trials. The absolute risk reduction enables calculation of the "number needed to treat" (NNT). The NNT is simply the inverse of the absolute risk reduction. ${ }^{3}$ The NNT represents the number of patients that need to be treated to prevent one adverse event.

\section{Relative risk}

Relative risk is the ratio of two absolute risks. It measures the strength of effect of an exposure (or treatment) on risk. ${ }^{2}$ A beneficial treatment will result in a relative risk of less than 1 ; this can then be subtracted from 1 to give the relative risk reduction. A harmful treatment, or other exposure, will give a relative risk of more than 1 .

In the British doctors study, over 40 years of follow up, the annual mortality rate from CHD was 572 per 100000 in non-smokers, and 892 per 100000 in smokers. ${ }^{4}$ For lung cancer the figures were 14 and 209, respectively. So the attributable risk of CHD related to smoking was 320 (892-572) per 100000 compared with 195 (209-14) per 100000 for lung cancer. These figures are the excess numbers of deaths (per 100 000) in smokers which were accounted for by their smoking. For an individual, smoking was much more likely to result in death from CHD than from lung cancer. The equivalent relative risks were 1.6 (892/572) for CHD and 14.9 (209/14) for lung cancer. So, smoking has a much stronger association with lung cancer than CHD (indicated by the larger relative risk). By contrast, smoking is associated with a larger attributable risk for CHD than for lung cancer. This is because CHD is a much more common condition than lung cancer among non-smokersthat is, the absolute risk is higher. So, a comparatively small increase in risk of CHD, associated with smoking (relative risk 1.6), results in a large number of deaths.

\section{Relative risk reduction in primary prevention}

If a condition is common the importance of reducing risk is much greater than if it is rare. In clinical practice, the relative risk reduction associated with treatment of blood pressure or serum cholesterol is constant at different levels of absolute risk. ${ }^{5}$ So, the absolute risk reduction associated with antihypertensive or cholesterol lowering treatment is proportional to the initial absolute risk. This is why guidelines for the primary prevention of CHD require quantitative prediction of absolute risk based on a patient's risk factor profile.

The Joint British Societies ${ }^{5}$ recommend that "as a minimum all individuals with an absolute CHD risk of 30\% or more over 10 years should be targeted now for comprehensive risk factor management, which will include, as appropriate, blood pressure and lipid lowering therapy" followed by "a progressive expansion of coronary prevention from $30 \%$ down to $15 \%$ absolute CHD risk". If we assume that the relative risk reduction associated with statin treatment is $33 \%$, in patients with an initial risk of $30 \%$ the absolute risk will be reduced (by one third) from $30 \%$ to $20 \%$ (that is, $10 \%$ ). In those with an initial risk of $15 \%$ it will be reduced (by one third again) from $15 \%$ to $10 \%$ (that is, only 5\%). These absolute risk reductions give NNTs of 10 and 20, respectively. So half as many patients with an initial risk of $30 \%$ (compared with $15 \%$ ) need to be treated to prevent one adverse event.

Other guidelines based on the same Framingham equations ${ }^{6}$ exist. ${ }^{7-10}$ However, in the recently published comparison study, in primary care, the joint British guidelines appeared to perform at least as well as others. ${ }^{11}$ Guidelines differ in their advice on the level of risk at which treatment should be initiated. In the UK the recommended threshold is partly an issue of cost to the National Health Service (NHS). The National service framework for coronary heart disease $^{12}$ recommends targeting risk reduction at those with a 10 year CHD risk greater than 30\%. This will mean 3\% of men aged $30-74$ years are targeted. ${ }^{5}$ If $15 \%$ were chosen as the threshold $28 \%$ would be eligible. So, while (initially) focusing on patients at highest risk sensibly targets resources at those who are most likely to benefit, the policy will deny a large proportion of the population effective treatment.

\section{Secondary prevention}

Of course, those patients with the greatest risk of a future CHD event are those with CHD already. Secondary prevention is therefore the area with the greatest potential for patient benefit. Absolute risk quantification is probably unnecessary in these patients, as all should be subject to attempts at risk reduction through: lifestyle changes (smoking, diet, and physical activity); the control of blood pressure, lipids, and glucose; and drug treatment (for example, aspirin). The purpose is to reduce the risk of a major cardiovascular event and reduce mortality. In these patients, it is important to realise and communicate the likely benefits of behavioural change in preventing further $\mathrm{CHD}$ events, such as the $50 \%$ relative risk reduction associated with stopping smoking, ${ }^{5}$ and the importance of long term compliance with antihypertensive and cholesterol lowering treatment, and aspirin, each of which are probably associated with relative risk reductions in CHD events of $10-30 \% .^{513}$ 
In order to initiate risk reduction strategies in $\mathrm{CHD}$, a clear understanding of the meanings and appropriate uses of absolute, attributable, and relative risk is required.

Department of Public Health Sciences,

JAMES E C SEDGWICK

Guy's, King's \& St Thomas' School of Medicine,

London SE1 3QD, UK

james.sedgwick@kcl.ac.uk

1 de Bono D. Complications of diagnostic cardiac catheterisation: results from 34,041 patients in the United Kingdom confidential enquiry into cardiac catheter complications. The joint audit committee of the British Cardiac Society and Royal College of Physicians of London. Br Heart $\mathcal{F}$ 1993;70:297-300.

2 Hennekens $\mathrm{CH}$, Buring JE. Epidemiology in medicine. Philadelphia: Lippincott Williams and Wilkins; 1987.

3 Sackett DL, Richardson WS, Rosenberg W, et al. Evidence based medicine: how to practice and teach EBM. Edinburgh: Churchill Livingstone, 1996.

4 Doll R, Peto R, Wheatley K, et al. Mortality in relation to smoking: 40 years' observations on male British doctors. BMF 1994;309:901-11.
5 British Cardiac Society, British Hyperlipidaemia Association, British Hypertension Society, endorsed by the British Diabetic Association. Joint Hypertension Society, endorsed by the British Diabetic Association. Joint British recommendations on prevention of
cal practice. Heart 1998;80 (suppl 2):S1-29.

6 Anderson KM, Odell PM, Wilson PW, et al. Cardiovascular disease risk profiles. Am Heart F 1991;121:293-8.

7 Ramsay LE, Haq IU, Jackson PR, et al. Targeting lipid-lowering drug therapy for primary prevention of coronary disease: an updated Sheffield table. Lancet 1996;348:387-8.

8 Wood D, de Backer G, Faergeman $\mathrm{O}$, et al, together with members of the Task Force. Prevention of coronary heart disease in clinical practice: recommendations of the second joint task force of European and other societies on coronary prevention. Atherosclerosis 1998;140:199-270.

9 Jackson R. Updated New Zealand cardiovascular disease risk-benefit prediction guide. BMF 2000;320:709-10

10 McCormack JP, Levine M, Rangno RE. Primary prevention of heart disease and stroke: a simplified approach to estimating risk of events and making drug treatment decisions. C Med Assoc f 1997;157:422-8.

11 Jones AF, Walker J, Jewkes C, et al. Comparative accuracy of cardiovascular risk prediction methods in primary care patients. Heart 2001;85:37-43.

12 Department of Health. National service framework for coronary heart disease. London: Department of Health, 2000 .

13 Antiplatelets Trialists Collaboration. Collaborative overview of randomised trials of antiplatelet therapy I. Prevention of death myocardial infarction and stroke by prolonged antiplatelet therapy in various categories of patients. BMF 1994;308:81-106.

\section{Right aortic arch and coarctation: delineation by three dimensional magnetic resonance angiogram}

A 14 year old girl returned for follow up. She initially presented with coarctation and right aortic arch at two months of age. Cardiac catheterisation determined the coarctation to be between the right common carotid (RCC) and right subclavian arteries (RSA), with a retro-oesophageal left subclavian artery. The intracardiac anatomy was normal. The patient underwent patch augmentation of the coarctation site at 2 months of age and again at 8 months of age for recurrent coarctation with a gradient of $80 \mathrm{~mm} \mathrm{Hg}$. An ascending aorta to the upper abdominal aorta conduit was placed at 15 months of age when she presented with recurrent coarctation. At 14 years of age cardiac catheterisation demonstrated a widely patent conduit but a residual coarctation between the right carotid and subclavian arteries with a $16 \mathrm{~mm} \mathrm{Hg}$ gradient. A magnetic resonance angiogram including three dimensional reconstruction provided excellent definition of the isolated coarctation

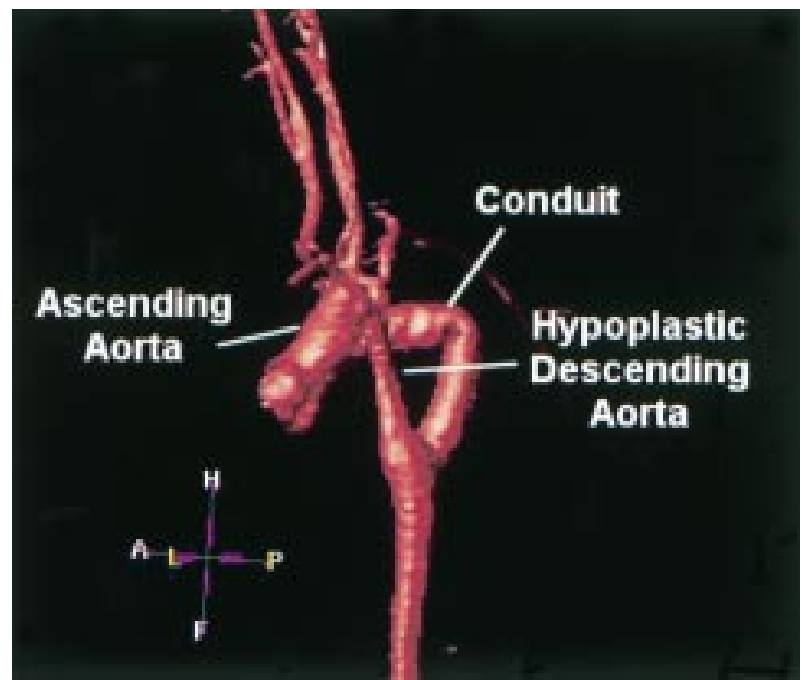

between the RCC and RSA, in addition to identifying additional areas of stenosis in the RSA and upper descending thoracic aorta (below left and right).

Right aortic arch in association with coarctation is extremely rare. This is in keeping with the principle of flow related development of the central great vessels. Coarctation is more likely to occur in situations of right to left shunting through the ductus arteriosus with decreased blood flow across the aortic isthmus. Additionally right aortic arch is strongly associated with right side obstructive lesions, in which there is reversed ductal flow and increased antegrade flow across the isthmus, which makes this entity even rarer.

COLIN J MCMAHON G WESLEY VICK III MICHAEL R NIHILL cmcmahon@bcm.tmc.edu

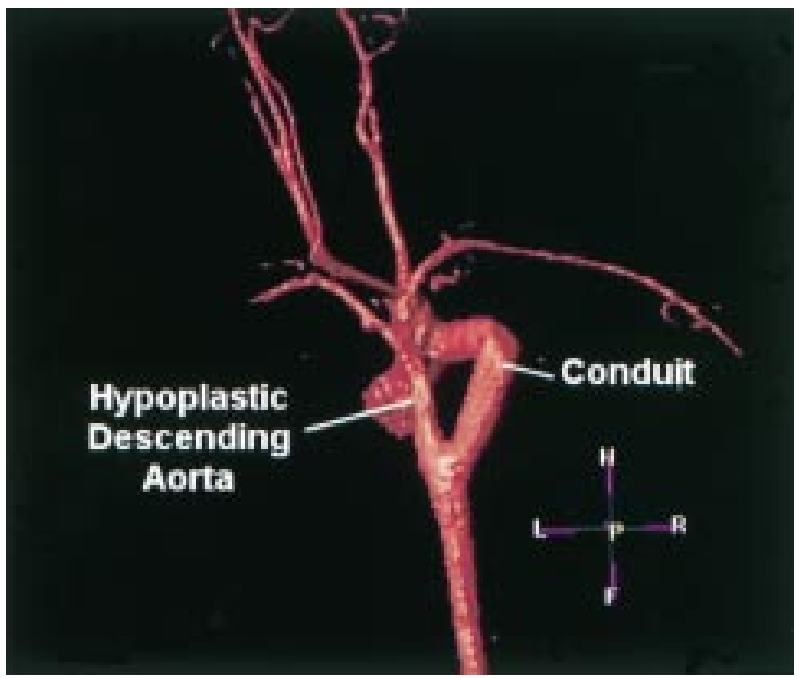

(Left) Left lateral view of three dimensional reconstructed gadolinium enhanced magnetic resonance angiogram showing the right aortic arch and the hypoplastic descending aorta. Note the residual coarctation between the right common carotid and subclavian arteries, and the additional areas of stenosis in the right subclavian artery and descending aorta. The mid-conduit stenosis can be clearly seen. There is a retro-oesophageal left subclavian artery. (Right) Posterior view showing the residual coarctation and the mid-conduit stenosis. A, anterior; P, posterior; $L$, left, $R$, right, $H$, head, $F$, foot. 